\title{
Parameter optimization of the OTFT gas sensor and its trace $\mathrm{NO}_{2}$-sensing properties
}

\author{
Yadong Jiang*, Huiling Tai, Xiaosong Du, Xian Li, Bo Zhang \\ State Key Laboratory of Electronic Thin Films and Integrated Devices, School of Optoelectronic \\ Information, University of Electronic Science and Technology of China (UESTC), Chengdu Jianshe \\ North Road, section2 No.4, 610054, P. R. China
}

\begin{abstract}
:
In this paper, the gas-sensing characteristics of gas sensors for Organic Thin-Film Transistors (OTFTs) with the different ratio of the channel length to width and thickness of the insulating layer were studied. The results showed that the device parameters had the influence on the sensitivity and response time of sensors, and the optimized parameters of OTFT sensors were obtained, that was, the ratio of the channel length to width was between 160 and 640, and the insulating layer was 195 $\mathrm{nm}$ in thickness. The OTFT sensor with the $\alpha$-Sexithiophene $(\alpha-6 \mathrm{~T})$ film incorporated into a sensitive layer was observed to respond strongly to the trace nitrogen dioxide $\left(\mathrm{NO}_{2}\right)$ vapor ranging from 0.2 to 1 ppm at room temperature.
\end{abstract}

Key words: gas sensors, OTFTs, device parameters, $\alpha-6 \mathrm{~T}, \mathrm{NO}_{2}$

\section{Introduction}

Using a bottom gate organic thin-film transistor (OTFT) as a gas sensor was put forward in the late 1980s, for the analyte-receptor binding strongly influence the source-drain current flowing across the transistor channel [1]. Furthermore, organic TFTs as gas sensors for electronic nose application were also investigated [2]. In the last several years much research effort to use OTFT as multi-parameter sensors have been devoted in pursuit of good levels of selectivity, reliability, and reproducibility at low cost. Organic semiconductor materials such as phthalocyanines, naphthalene tetracarboxylic derivatives, pentacene, oligothiophenes, poly(phenylene ethynylene) are mainly used for the active layer [3-10]. Responses of OTFT sensors are simply noted as changes in the output source-drain current for a given set of input drain voltages and gate voltages as the vapor adsorbs onto the OTFT [3].

In this manuscript, we reported initial studies of $\alpha$-Sexithiophene ( $\alpha-6 \mathrm{~T})$ film OTFT interacting with the trace nitrogen dioxide $\left(\mathrm{NO}_{2}\right)$ gases $(0-1$ $\mathrm{ppm}$ ). The effect of the device parameter on the vapor-sensing properties, such as the ratio of the channel length to width and the thickness of the insulating layer, were measured and discussed.

\section{Experimental}

Fig. 1 shows the schematic illustration of bottomgated, bottom-contacting OTFT sensors. For the different ratio of the channel length to width, the thickness of the dielectric layer was fixed as 195 $\mathrm{nm}$. The channel length was $25 \mu \mathrm{m}$, and channel widths were $1000 \mu \mathrm{m}, 4000 \mu \mathrm{m}$ and $16000 \mu \mathrm{m}$. That was, the ratio of the channel length to width (W/L) was 40, 160 and 640, respectively. In addition, the influence of the insulating layer thickness on the gas-sensing characteristics was also studied, and $125 \mathrm{~nm}, 195 \mathrm{~nm}$ and 300 $\mathrm{nm}$ of $\mathrm{SiO}_{2}$ were thermally grown on heavily Sbdoped silicon wafers.

The $\alpha$-Sexithiophene ( $\alpha-6 T$ ) was purchased from $\mathrm{TCl}$ Development Co.Ltd, and the copper phthalocyanine (CUPc) was purchased from Luminescence Technology Corp. Both of them were used without any further purification. The a-6T was deposited by vacuum deposition as the sensitive layer for the detection of $\mathrm{NO}_{2}$, and the CuPc thin film was chosen as the active layer for the test of hydrogen sulfide $\left(\mathrm{H}_{2} \mathrm{~S}\right)$. All the sensitive thin film was about $90 \mathrm{~nm}$.

The current-voltage characteristics of the prepared device were studied using a Keithley 4200-SCS source measurement unit. As the pchannel transistor, the current-voltage (I-V) curves of the OTFT device were measured in the accumulation mode. The saturation sourcedrain currents were recorded as the output signals of sensors. All the measurement results 
are obtained at room temperature in $\mathrm{N}_{2}$ atmosphere. Gas concentration is controlled by a mass flow controls (MT50-4J, Beijing Metron Instruments Co.Ltd., China).

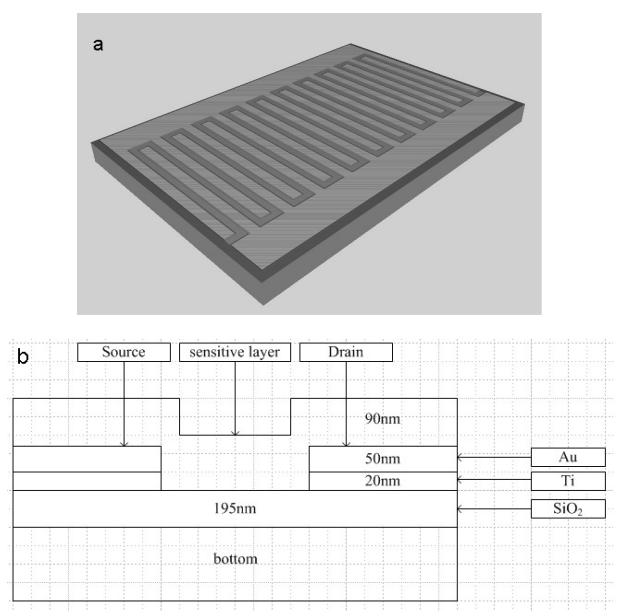

Fig. 1. Schematic illustration of the bottom-gated, bottom-contacting OTFT sensor (a) three-dimensional and (b) planar diagram

The surface morphology was observed with a Hitachi $\$ 4800$ scanning electronic microscope (SEM).

\section{Microstructure Characterization}

Fig. 2 and Fig. 3 show the SEM images of the $\alpha-$ 6T and CuPc sensitive films deposited on ITO glass, respectively. The surface morphology of the films was seen to be dense and crack-free.

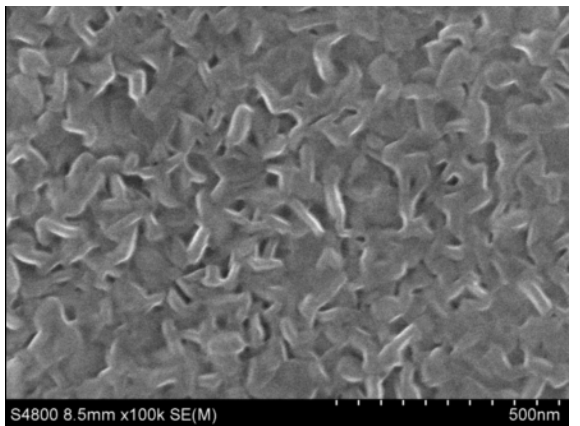

Fig. 2. The SEM micrograph of the $\alpha-6 T$ thin film deposited on ITO glass

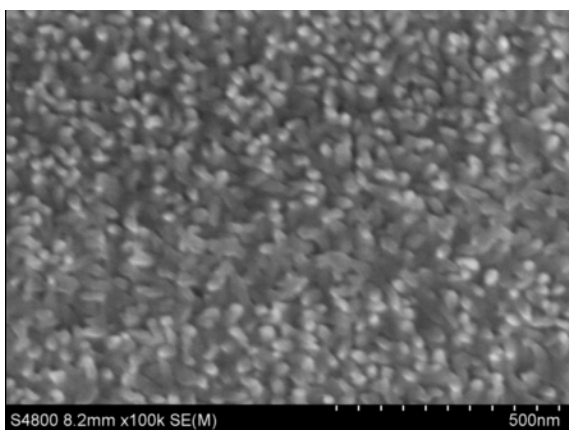

Fig. 3. The SEM micrograph of the CUPC thin film deposited on ITO glass

\section{Electronic characteristics of OTFT}

Fig.4(a) shows the typical drain current $\left(I_{d s}\right)$ and drain voltage $\left(V_{d s}\right)$ characteristics at different gate biases of bottom contact $\alpha-6 T$ OTFT $(\mathrm{W} / \mathrm{L}=640)$ operating in the accumulation mode (gate negatively biased). The $V_{d s}$ swept from 0 to $-60 \mathrm{~V}$ at gate bias $\left(V_{g s}\right)$ between 0 to $-60 \mathrm{~V}, I_{d s}$ increases almost linearly at low $V_{d s}$ values and then tends to saturate at higher $V_{d s}$ values [4-8]. And Fig.4(b) shows transfer characteristics of OTFTs at $V_{d s}=-50 \mathrm{~V}$.
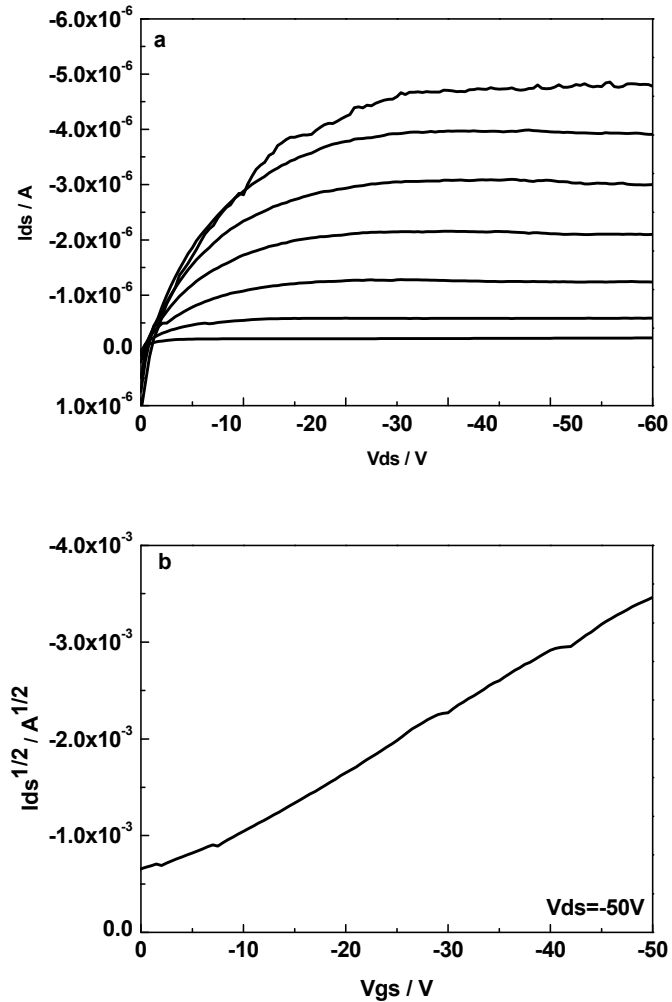

Fig. 4. The electronic characteristics of $\alpha-6 T$ OTFT (a)Drain current $\left(I_{d s}\right)$ vs drain voltage $\left(V_{d s}\right)$ characteristics at various gate voltages $\left(V_{g s}\right) ;(b) I_{d s}{ }^{1 / 2}$ vs $V_{\text {gs }}$ characteristics $\left(V_{d s}=-50 \mathrm{~V}\right)$

The $I_{d s}$ in the linear and saturation regions is given by eq.(1) and (2), respectively:

$$
\begin{aligned}
& I_{d s}=\frac{W C_{i} \mu}{L}\left[\left(V_{g s}-V_{t h}\right) V_{d s}-\frac{1}{2} V_{d s}^{2}\right] \\
& I_{d s a t}=\frac{W C_{i} \mu}{2 L}\left(V_{g s}-V_{t h}\right)^{2}
\end{aligned}
$$

Here, $W$ and $L$ is the channel width and length, respectively. $C_{i}$ is the capacitance of the insulator, $\mu$ is the carrier mobility, and $V_{t h}$ is the so-called threshold voltage that accounts for any voltage drop at the insulator semiconductor interface and the bulk conductivity of the semiconductor layer. $C_{i}$ is given by eq.(3): 


$$
C_{i}=\frac{\varepsilon_{i} \varepsilon_{0}}{d}
$$

Here, $d$ is the thickness of insulator layer, $\varepsilon_{i}$ and $\varepsilon_{0}$ are the dielectric constant and capacitance of vacuum, respectively.

\section{Parameter optimization of OTFT sensors}

The sensor sensitivity (S) is defined as $\left(\lg -I_{N}\right) / I_{N}$, where Ig and $I_{N}$ are the saturation drain-source current of the OTFT gas sensor in the measuring gas and nitrogen, respectively. And the response time is defined as the time required reaching $90 \%$ total current change. The dynamic response of $\alpha-6 \mathrm{~T}$ thin film OTFT sensor with the different ratio of $\mathrm{W} / \mathrm{L}$ exposed to $2 \mathrm{ppm}$ $\mathrm{NO}_{2}$ was investigated at room temperature, and the result was displayed in Fig.5. It is noticeable that the response time and recovery time were limited to $600 \mathrm{~s}$ for the convenience of comparison. And the sensitivity and the response time can be calculated from the graph curve of Fig.5, which was shown in Tab.1. It can be seen that the largest sensitivity of the OTFT sensor was obtained with the ratio of W/L as 640 , which also had the longest response time as $300 \mathrm{~s}$. Therefore, it was speculated that the ratio of the W/L should be between 160 and 640 .

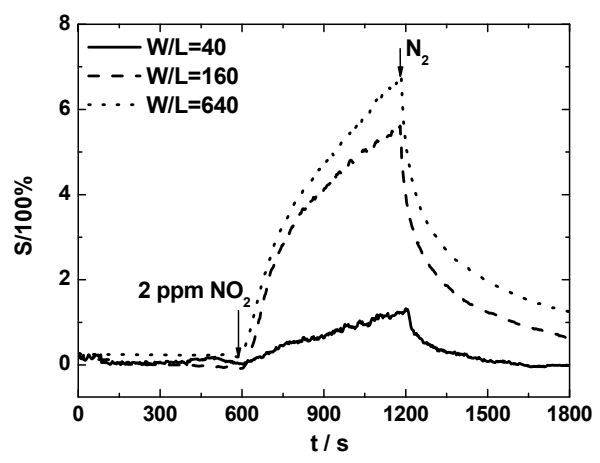

Fig.5. Response transients of a-6T OTFT sensor with the different ratio of $W / L$ exposed to $2 \mathrm{ppm} \mathrm{NO}_{2}$

Tab. 1: The sensitivity and the response time of $\alpha-6 T$ OTFT sensor with the different ratio of W/L exposed to $2 \mathrm{ppm} \mathrm{NO}$

\begin{tabular}{|c|c|c|}
\hline W/L & Sensitivity & Response time (s) \\
\hline 40 & 1.32 & 130 \\
\hline 160 & 5.57 & 206 \\
\hline 640 & 6.72 & 300 \\
\hline
\end{tabular}

The response and recovery curves of OTFT samples with the distinct thickness of dielectric layer exposed to $200 \mathrm{ppm} \mathrm{H}_{2} \mathrm{~S}$ were plotted as a function of time in Fig.6, and the corresponding sensing values were listed in Tab.2, indicating that the OTFT sensor composed of dielectric layer of $195 \mathrm{~nm}$ had the optimized gas-sensing property.

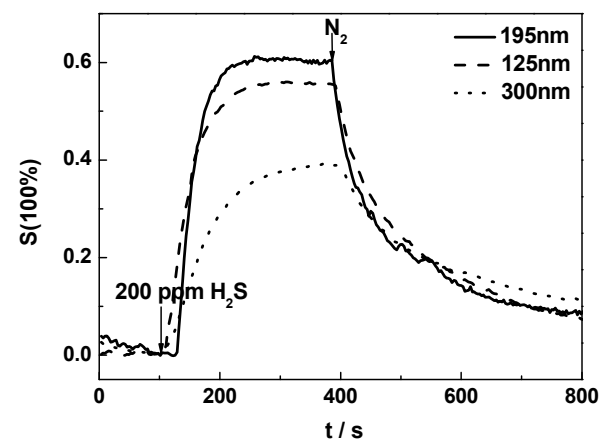

Fig. 6. Response transients of OTFT sensor with the different thickness of the dielectric layer exposed to $200 \mathrm{ppm} \mathrm{H}_{2} \mathrm{~S}$

Tab. 2: The sensitivity and the response time of $\alpha-6 T$ OTFT sensor with the different ratio of $\mathrm{W} / \mathrm{L}$ exposed to $2 \mathrm{ppm} \mathrm{NO}$

\begin{tabular}{|c|c|c|}
\hline$T_{\text {SiO2 }}$ & Sensitivity & Response time (s) \\
\hline 125 & 0.56 & 86 \\
\hline 195 & 0.61 & 65 \\
\hline 300 & 0.39 & 141 \\
\hline
\end{tabular}

$T_{\text {SiO2: }}$ The thickness of the $\mathrm{SiO}_{2}$ layer.

\section{Trace $\mathrm{NO}_{2}$-sensing properties}

Based on the above parameter optimization of OTFT sensors, the trace $\mathrm{NO}_{2}$-sensing properties were further measured and discussed employing the OTFT sensor with the ratio of W/L as 320 and the thickness of the dielectric layer as 195 $\mathrm{nm}$, using the $\alpha-6 \mathrm{~T}$ thin film as the sensitive thin film. It can be seen that the drain-source current increases dramatically after exposed to $\mathrm{NO}_{2}$ gas, which might be ascribed to the adsorption effect between $\mathrm{NO}_{2}$ gas molecules and the polarbonding of $\alpha-6 T$ thin film. Also, the good reproducibility and reversibility were indicated.

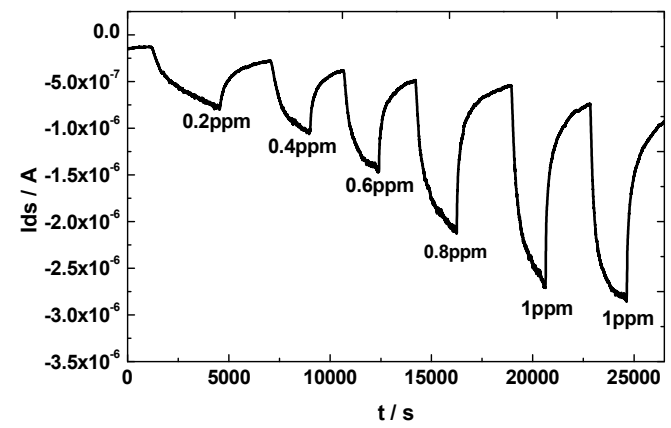

Fig. 7. The change in response as a function of time when the OTFT sensor was exposed to cycles of exposure and evacuation of $\mathrm{NO}_{2}$ concentration ranging from 0.2 to $1 \mathrm{ppm}$ at room temperature 


\section{Conclusion}

The sensing properties of OTFTs with different ratio of channel width to length and the thickness of dielectric layer were studied. The results showed that OTFTs with the W/L between 160 and 640 and the $195 \mathrm{~nm}$ dielectric layer provided the optimized gas-sensing properties. The OTFTs based on $\alpha-6 T$ sensitive film can be used for the trace $\mathrm{NO}_{2}$ gas detection, and the least detection limit is $0.2 \mathrm{ppm}$.

\section{Acknowledgements}

This work was supported by National Science Foundation of China via grants No.61101031 and No.61101029, and the Fundamental Research Funds for the Central Universities via grants No. ZYGX2009J052.

\section{References}

[1] A. Tsumura, H. Koezuka, K. Ando, Macromolecular electronic device: Field-effect transistor with a polythiophene thin film, Applied Physics Letter, 49, 1210-1212(1986), doi: 10.1063/1.97417

[2] F. Liao, C. Chen, V. Subramanian, Organic TFTs as gas sensors for electronic nose applications, Sensors and Actuators B, 107, 849-855 (2005); doi: 10.1016/j.snb.2004.12.026

[3] J. Huang, J. Miragliotta, A. Becknell, H. E. Katz, Hydroxy-Terminated Organic SemiconductorBased Field-Effect Transistors for Phosphonate Vapor Detection, Journal of the American Chemical Society, 129, 9366-9376 (2007); doi: $10.1021 / \mathrm{ja0689} 64 \mathrm{z}$

[4] J.B. Chang, V. Liu, V. Subramanian, K. Sivula, C. Luscombe, A. Murphy, J. Liu, M.J. Frechet, Printable polythiophene gas sensor array for lowcost electronic noses, Journal of Applied Physics, 100,014506 (2 0 06); doi:10.1063/1.2208743

[5] L. Torsi, F. Marinelli, M. D. Angione, A. D. Aquila, N. Cioffi, E.D. Giglio, L. Sabbatini, Contact effects in organic thin-film transistor sensors, Organic Electronics, 10, 233- 239(2009); doi:10.1016/j. orgel. 2008.11.009

[6] L. Torsi, A. Dodabalapur, L. Sabbatini, P.G. Zambonon, Multi-parameter gas sensors based on organic thin-film-transistors, Sensors and Actuators B, 67, 312-316 (2000); doi: 10.1016/ S0925-4005(00)0054 1-4

[7] L. Torsi, M. C. Tanese, N. Cioffi, M. C. Gallazzi, L. Sabbatini, P. G. Zambonin, G. Raos, S. V. Meille, M. M. Giangregorio, Side-Chain Role in Chemically Sensing Polymer Field-Effect Transistors, The Journal of Physical Chemistry B, 107, 7589-7594(2003); doi: 10.1021/jp03449 51

[8] L. Torsi, A. Dodabalapur, Organic Thin-Film Transistor as Plastic Analytical Sensors, Analytical Chemistry, 77, 380A-387A (2005), doi:10.1021/ ac053475n
[9] J. Liu, M. Agarwal, K. Varahramyan, Glucose sensor based on organic thin film transistor using glucose oxidase and conducting polymer, Sensors and Actuators B, 135, 195-199 (2008); doi: 10.1016/j.snb.2008.08.009

[10] X. Li, Y. Jiang, G. Xie, X. Du, H. Tai, J. Yan, S. Fu, Ethanol gas sensors based on copper phthalocyanine thin-film transistors, Apperceiving Computing and Intelligence Analysis (ICACIA), 2010 International Conference, 470-473, doi:10.1109/ICACIA.2010.5709943 